HIGH TEMPERATURE POLYMER DIELECTRIC FILM INSULATION

Robert J. Jones

TRW

Redondo Beach, California

N94- 28715

HIGH TEMPERATURE POLYMER DIELECTRIC FILM INSULATION

\title{
BACKGROUND
}

- DUAL USE SYSTEM DRIVERS FOR DEVELOPMENT, QUALIFICATION AND PRODUCTION OF NEXT

GENERATION, VERY HIGH TEMPERATURE WIRE INSULATION

- ENERGY RECOVERY/GENERATION/DISTRIBUTION AND TRANSPORTATION MARKETS (1990'S)

- SUPER CAPACITY/RESPONSE ELECTRONIC COMPUTATION AND TELECOMMUNICATION EQUIPMENT (1990's)

- HIGH SPEED CIVIL TRANSPORT (NEXT CENTURY)

- ALl ELECTRIC AIRPLANe (NEXT CENTURy)

- EXAMPLES OF SYSTEM RATIONALE FOR VERY HIGH TEMPERATURE WIRE INSULATION

- SMALLER, MORE EFFICIENT ELECTRONIC SYSTEMS RUN HOTTER

- OPERATING ENVIRONMENTS SUCH AS DOWNWELL ARE GETTING MORE THERMALLY SEVERE

- ACTIVE COOLING SYSTEMS FOR GENERATORS/ALTERNATORS, STORAGE/TRANSHISSION/ DISTRIBUTION SYSTEMS AND BLACK BOXES ARE COSTLY ANO EQUATE TO SEVERE WEIGHT PENALTIES

- SMALLER DIAMETER WIRES MAY BE SUFFICIENT TO CARRY EQUIVALENT POWER

\section{HIGH TEMPERATURE POLYMER DIELECTRIC FILM INSULATION}

\section{BACKGROUND (CONTINUED)}

- ASSESSMENT OF EMERGING REQUIREMENTS HAS DICTATED THAT 300 C PERFORMANCE IS THE GOAL FOR NEXT GENERATION WIRE INSULATION

- VERY SIGNIFICANT INCREASE OVER CURRENTLY QUALIFIED POLYIMIDE AND FLUOROPOLYMERS RATED AT $200^{\circ} \mathrm{C}$ (OR SLIGHTLY ABOVE)

- EMERgINg hIGH TEMPERATURE POLYMER MATERIALS HAVE BEEN SHOWN TO HAVE POTENTIAL FOR PERFORMANCE AT $\geq 300^{\circ} \mathrm{C}$

- $\quad \geq 300^{\circ} \mathrm{C}$ INSULATIONS SHOULD MEET NEW DUAL USE PERFORMANCE REQUIREMENTS WELL INTO NEXT CENTURY

- STATUS OF RECENT OR CURRENT $300^{\circ} \mathrm{C}$ POLYMERIC WIRE INSULATION ACTIVITY

- UBE INDUSTRIES OFFERED UPILEX ${ }^{R}$ FILM, BUT WITHDREW IT FRON THE MARKET IN 1992

- FOSTER MILLER IS STUDYING LIQUIO CRYSTAL POLYMERS

- 3M IS DEVELOPING FPE POLYMER MATERIAL

- TRW HAS SHOWN HIGH PROMISE FOR ITS PFPI POLYMERS UNDER USAF SPONSORSHIP (FINAL REPORT WL-TR-91-2105); FURTHER WORK WILL BE CONDUCTED IN RECENTLY AWARDED USAF CONTRACT F33615-93-C-2367 
- REPRESENTATIVE CHEMISTRY<smiles></smiles>

US PATENT NUMBERS $4,111,906 ; 4,196,277 ; 4,203,922 ; 4,880,584$

(PFPI POLYMERS WERE INVENTED UNDER NASA LEWIS RESEARCH CENTER SPONSORSHIP IN THE LATE 1970's)

- VERSATILITY

- FORMULATIONS CAN BE TAILOREd TO MEET PRODUCT USE REQUIREMENTS

- COATING VARNISH, FILM AND POWDER PRODUCT FORMS CAN BE EMPLOYED TO ADAPT FORMULATIONS TO EXISTING PROCESSING EQUIPMENT FOR CONVERSION TO WIRE INSULATION

- POLYMERS POSSESS SUPERIOR COMBINATION OF THERMAL/ELECTRICAL/UV, MOISTURE \& FLUID RESISTANCE/TRIBOLOGICAL PROPERTIES

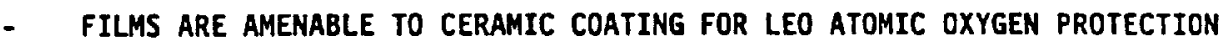

\section{HIGH TEMPERATURE POLYMER DIELECTRIC FILM INSULATION}

\section{TRW PFPI AS SUPERIOR $300^{\circ} \mathrm{C}$ POLYMER CAMDIDATES (CONTINUED)}

- COMPARISON OF PROMISING PFPI FILM PROPERTIES WITH KAPTON (FROM REPORT WL-TR-91-2105)

\begin{tabular}{|c|c|c|}
\hline \multirow[b]{2}{*}{ PROPERTY MEASURED } & \multicolumn{2}{|c|}{ PROPERTY RESULT $\left.{ }^{A}\right)$} \\
\hline & KAPTON FILM & TRH PFPI FILM \\
\hline $\begin{array}{l}\text { EIECIRICAL } \\
\text { - DIELECTRIC CONSTANT } \\
- \text { AT } 25^{\circ} \mathrm{C} \\
- \text { AT } 300^{\circ} \mathrm{C}\end{array}$ & $\begin{array}{l}3.1 \\
2.8\end{array}$ & $\begin{array}{l}3.1 \\
2.9\end{array}$ \\
\hline $\begin{array}{l}\text { - OISSIPAIION FACTOR } \\
- \text { AT } 25^{\circ} \mathrm{C} \\
- \text { AT } 300^{\circ} \mathrm{C}\end{array}$ & $\begin{array}{l}0.001 \\
0.063\end{array}$ & $\begin{array}{l}0.001 \\
0.004\end{array}$ \\
\hline $\begin{array}{l}\text { - BREAKDOWN VOLTAGE AT } 25^{\circ} \mathrm{C} \text { (V/MIL) } \\
=\text { AC } \\
=\text { OC }\end{array}$ & $\begin{array}{r}7000 \\
11000\end{array}$ & $\begin{array}{r}6000 \\
12000\end{array}$ \\
\hline $\begin{array}{l}\text { LOW TEMPERATURE STABILITY (CRYOGENIC) } \\
\text { EXPOSURE IN LIQUID NITROGEN AND } \\
\text { HELIUM }\end{array}$ & NO EFFECT & NO EFFECT \\
\hline $\begin{array}{l}\text { AIR AGING AT } 300^{\circ} \mathrm{C} \\
\text { - MEIGHT LOSS AFTER } 1000 \text { HRS (x) }\end{array}$ & 13.0 & 4.1 \\
\hline $\begin{array}{l}\text { HUMIDITY AGING AI } 90^{\circ} \mathrm{C} / 100 \% \text { RH } \\
\text { - WEIGHT LOSS AFTER } 1200 \text { HRS }(\%)\end{array}$ & FAILED AFTER 500 HRS & 0.4 \\
\hline $\begin{array}{l}\text { BASIC SOLUTION (PH, 10) AGING AT } 93^{\circ} \mathrm{C} \\
\text { WEIGHT LOSS AFTER } 96 \text { HRS (x) }\end{array}$ & 2.6 & 1.3 \\
\hline 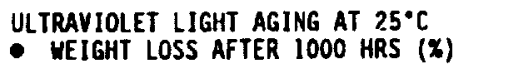 & 6.7 & 1.4 \\
\hline
\end{tabular}

A) ALL PROPERTIES DETERMINED ON 0.001-INCH THICK FILMS 


\section{TRW'S PFPI AS SUPERIOR $300^{\circ} \mathrm{C}$ POLYMER CANDIDATES (CONTINUED)}

- PROMISING BULK POLYMER OR COATING PROPERTIES

\begin{tabular}{|c|c|c|}
\hline PROPERTY TYPE & PROPERTY MEASURED & TEST RESULT \\
\hline THERMAL & $\begin{array}{l}\text { - MELTING POINT } \\
\text { - GLASS TRANSITION } \\
\text { TEMPERATURE }\end{array}$ & $\begin{array}{l}2400^{\circ} \mathrm{C} \\
>300^{\circ} \mathrm{C}\end{array}$ \\
\hline TRIBOLOGICAL & $\begin{array}{l}\text { - friction coefficient } \\
\text { - wear rate }\end{array}$ & $\begin{array}{l}0.3-0.6(R T) ; 0.1-0.2\left(300^{\circ} \mathrm{C}\right) \\
\text { MUCH LOWER THAN TEFLON }\end{array}$ \\
\hline $\begin{array}{l}\text { COATING } \\
\text { ENVIRONMENTAL RESISTANCE }\end{array}$ & $\begin{array}{l}- \text { COATING INTEGRITY } \\
\text { AFTER EXPOSURE TO: } \\
-500 \text { HRS, } 343^{\circ} \mathrm{C} \\
-21 \text { DAYS, } 71^{\circ} \mathrm{C} \text { IN } \\
\text { MIL-H-5606 } \\
\text { HYDRAULIC FLUID } \\
-21 \text { DAYS, } 71^{\circ} \mathrm{C} \text { IN } \\
\text { MIL-L-7808 JET } \\
\text { ENGINE OIL } \\
\text { - } 2000 \text { HRS, } 25^{\circ} \mathrm{C} \\
\text { IN 5X SALT SPRAY }\end{array}$ & $\begin{array}{l}\text { NO } \\
\text { BLISTERING } \\
\text { OR } \\
\text { LOSS } \\
\text { OF } \\
\text { AOHESION }\end{array}$ \\
\hline
\end{tabular}

HIGH TEMPERATURE POLYMER DIELECTRIC FILM INSULATION

TRW'S PFPI AS SUPERIOR $300^{\circ} \mathrm{C}$ POLYMER CAMDIDATES (CONTINUED)

- duAl USE ChALLENGes fOR 1994-1996 TIME fRAME

- CONTINUING USAF WORK

- VERIFY PROMISING INITIAL FILM AND COATING PROPERTIES AS A WIRE INSULATION

- DEMONSTRATE A SUPERIOR $300^{\circ} \mathrm{C}$ AOHESIVE FOR WRAPPED FILM

- ACHIEVE HIGH INSULATION RESISTANCE TO ARCING \& TRACKING

- ACHIEVE FACILE FILM WRAP PROCESSABILITY ON EXISTING PLANT EQUIPMENT AND PRODUCE HIGH QUALITY INSULATED WIRE

- COMMERCIAL PRODUCT DEVELOPMENT

- QUALIFY AND INTRODUCE PFPI INTO MAGNET WIRE INSULATION, AUTOMOTIVE COMPONENT COATING AND MEDICAL DIAGNOSTIC PRODUCT APPLICATIONS

- MAXIMIZE HIGH VOLUME USE APPLICATIONS TO MINIMIZE FUTURE POLYMER COSTS

- PROPOSED ADAPTATION OF PFPI TO MEET FUTURE NASA $200^{\circ} \mathrm{C}$ SPACE SYSTEM WIRE INSULATION REQUIREMENTS

- OETERMINE INITIAL BASELINE MIRE PROPERTIES SPECIFIC TO SPACE APPLICATION ON COATED OR WRAPPED WIRE

- TAILOR EXISTING $300^{\circ} \mathrm{C}$ POLYMER CANDIDATE TECHNOLOGY, AS REQUIRED, TO OFFER OPTIMUM $200^{\circ} \mathrm{C}$ PERFORMANCE; BUILD UPON EURECA SAMPLE TEST RESULTS

- PROOUCE OPTIMIZED INSULATED WIRE AND PERFORM QUALIFICATION TESTS FOR GENERAL AND MISSION SPECIFIC SPACE APPLICATIONS 
